\title{
Testing Planck-Scale Gravity with Accelerators
}

\author{
Vahagn Gharibyan* \\ Deutsches Elektronen-Synchrotron DESY - D-22603 Hamburg, Germany
}

(Received 1 August 2012; published 4 October 2012)

\begin{abstract}
Quantum or torsion gravity models predict unusual properties of space-time at very short distances. In particular, near the Planck length, around $10^{-35} \mathrm{~m}$, empty space may behave as a crystal, singly or doubly refractive. However, this hypothesis remains uncheckable for any direct measurement, since the smallest distance accessible in experiment is about $10^{-19} \mathrm{~m}$ at the LHC. Here I propose a laboratory test to measure the space refractivity and birefringence induced by gravity. A sensitivity from $10^{-31} \mathrm{~m}$ down to the Planck length could be reached at existent $\mathrm{GeV}$ and future $\mathrm{TeV}$ energy lepton accelerators using laser Compton scattering. There are already experimental hints for gravity signature at distances approaching the Planck length by 5-7 orders of magnitude, derived from SLC and HERA data.
\end{abstract}

DOI: 10.1103/PhysRevLett.109.141103

PACS numbers: 04.80.Cc, 04.60.-m, 29.27.Hj

Introduction.-The quantum formalism cannot be directly applied to gravitation, and that is one of the major problems on a way of understanding and describing the physical reality. An important reason for this is the dynamical space concept adopted in general relativity, the currently accepted theory of gravity which states that any mass or particle modifies the space geometry (or metrics). On the other hand, the successful quantum theories within the Standard Model operate only in a fixed geometry space. For instance, observed violations of the discrete symmetries such as space, charge, and time parities are attributed to the particles and their interactions, while the scene of the interactions, the space-time, is considered to remain perfectly symmetric [1]. These two faces of space are believed to unify at distances near the Planck length $l_{P}=$ $1.6 \times 10^{-35} \mathrm{~m}$ (or mass $M_{P}=1.2 \times 10^{19} \mathrm{GeV}$, natural units are assumed throughout the Letter). At this scale gravity is expected to be similar in strength to the electroweak and strong forces, and quantum effects become important for the gravitational field. String theory and loop quantum gravity theory are prominent candidates which set a framework to make predictions in that energy domain. In many cases, unconventional space-time properties are suggested, such as vacuum refractivity [2] and/or birefringence [3]. As suggested in this Letter, such effects may be studied by using high energy accelerator beams. The proposed experiments probe the vacuum symmetry in a search for a handedness or chirality of the empty space presumed by quantum gravity. One particular example is circular birefringence $\Delta n=n_{L}-n_{R}$ of space, with $n_{L(R)}$ being the refraction index of left (right) helicity photons traversing the space. In the following, quantitative theoretical estimates and existing experimental limits are quoted, the formalism of the proposed method is presented, and the sensitivity of accelerator experiments is discussed.

Quantum and torsion gravity predictions.-Since Planck mass $M_{P}=\sqrt{c \hbar / G}$ is built from the speed of light and fundamental Planck and gravitational constants, this mass scale is considered to be relativistic and quantum gravitational. Most general modification of photon dispersion relation at lowest order of Planck mass could be expressed as

$$
\omega^{2}=k^{2} \pm \xi \frac{k^{3}}{M_{P}},
$$

where $\omega$ and $k$ are the photon's energy and momentum, respectively, while the $\xi$ is a dimensionless parameter and the \pm signs stand for opposite helicity photons [3].

Several theories are predicting or supporting the relation (1). The Planck-scale quantum gravity modifies the Maxwell equations by adding extra terms proportional to the Planck length [4]:

$$
\begin{gathered}
\frac{\partial \vec{E}}{\partial t}=\vec{\nabla} \times \vec{B}-2 \xi l_{P} \vec{\nabla}^{2} \vec{B}, \\
\frac{\partial \vec{B}}{\partial t}=-\vec{\nabla} \times \vec{E}-2 \xi l_{P} \vec{\nabla}^{2} \vec{E},
\end{gathered}
$$

which leads to a deformed energy-momentum or dispersion relation (1). In the above equations, $\vec{E}$ and $\vec{B}$ describe the electromagnetic field. More general expressions accounting for space anisotropy are derived in Ref. [5]. Using conventional definition $n=d \omega / d k$, it is easy to verify that Eqs. (1)-(3) introduce a chiral vacuum with an energy dependent birefringence

$$
\Delta n=3 \times 10^{-19} \xi \omega[\mathrm{GeV}],
$$

where the magnitude of $\xi$ defines the characteristic energies or distances where quantum-gravity effects become sizable. In the simplest possible picture, this only happens at the Planck scale, and hence $\xi=1$. However, the running of fundamental constants with energy may require quantum gravity to become active a few orders of magnitude below the Planck scale. The parameter $\xi$ is there to account for such effects. 
Another possible source of vacuum chirality is described by torsion gravity, an extension of the general relativity into the microscopic world to include particles' spins-for a review see [6]. In general, the spin gravity (space torsion) is considered to be weaker than the mass gravity (space curvature). However, near the Planck scale it may become detectable. Following Ref. [7], from the electromagnetic field Lagrangian

$$
\mathcal{L}=-\frac{1}{4} F_{\mu \nu} F^{\mu \nu}+q T^{\mu \nu \rho}\left(\partial^{\sigma} F_{\mu \nu}\right) F_{\rho \sigma}
$$

with a torsion tensor $T^{\mu \nu \rho}$ and free parameter $q$ one derives a dispersion relation quite similar to Eq. (1):

$$
\omega^{2}=k^{2} \pm q S_{0} k^{3},
$$

where $S_{0}$ stands for a time component of the contorsion vector.

Myers and Pospelov [8] derived the expression (1) within effective field theory with dimension 5 operators. A similar effect is calculated in Ref. [9] exploring graviton interaction with an electromagnetic field in one-loop approximation. In summary, chiral space is a universal feature of Planck-scale gravity, in the sense that it is predicted by a large diversity of theories.

A nonbirefringent gravitational space is also possible and has been predicted within string theories using $D$-brane formalism. In Ref. [10], a polarization independent refractivity

$$
n-1=\zeta \frac{k}{M_{P}}
$$

is obtained for the space-time foam near the Planck length. Here we use $\zeta$ instead of the $\xi$ to distinguish between the nonchiral and chiral space. In principle, both types may occur in the same vacuum at different scales $\zeta$ and $\xi$. Both gravity induced effects, namely, birefringence and refractivity, share the common feature that their strength is growing with the photon energy. This is in contrast to the usual condensed matter or electromagnetic, nontrivial vacua where the refraction effects are suppressed by powers of the energy $[11,12]$.

Current limits.-Experimental limits on space chirality are set by astrophysical observations exploring birefringence induced depolarization of the linear light which comes from distant cosmological sources [13]. The limits, however, are based on assumptions about the origin, spatial or temporal distribution of the initial photons, and their possible interactions during the travel. Another critical assumption is a uniformly distributed birefringence over cosmological distances. The most stringent limit $\xi<$ $2.4 \times 10^{-15}$ is set by Ref. [14] based on photons with polarization $0.63 \pm 0.30$ in an energy range from 100 to $350 \mathrm{keV}$ from GRB041219a [15]. Sensitive particlephysics effects have been suggested to test quantum gravity, mainly using threshold energies [16]. Applying cosmic ray constraints on photon decay and vacuum Cherenkov radiation [17], one arrives to $\zeta<30$ and $\zeta<300$ limits, respectively.

For the space refractivity, there are astrophysical observations interpreted [18] as $\zeta \sim 10$. This is derived from energy dependent time delay measurements of photons from distant sources. Similar to the results derived from polarized photons of cosmological origin, strong assumptions have to be made on the source of these photons. In addition, quoted astrophysical constraints are valid only for photon-virtual graviton loop interactions, since the photon path is essentially free from gravitational fields.

Terrestrial measurement could shed light on the quantum-gravity promoted space chirality and refractivity including effects introduced by real, Earth gravitons. In the laboratory the Planck scale can be accessed by exploring the extreme sensitivity of the high energy Compton scattering to the vacuum refraction as discussed in the following.

Compton scattering affected by gravity.-Let us denote by $\omega_{0}, \omega, \theta_{0}$, and $\theta$ the energies and angles of the incident and scattered photons relative to the initial electron direction. Then, according to Ref. [17], for the high energy Compton scattering in a vacuum with $n \approx 1$ (up to $\mathcal{O}\left[(n-1)^{2}\right]$ terms), the energy-momentum conservation yields

$$
n-1=\frac{\mathcal{E}}{2 \gamma^{2}(\mathcal{E}-\omega)}\left(1+x+\theta^{2} \gamma^{2}-x \frac{\mathcal{E}}{\omega}\right),
$$

where $\gamma$ and $\mathcal{E}$ are the Lorentz factor and energy of the initial electron, $x \equiv 4 \gamma \omega_{0} \sin ^{2}\left(\theta_{0} / 2\right) / \mathrm{m}$, and $n$ is the refraction index for the direction $\theta$ and energy $\omega$. This formula is more general than Eq. (3) of Ref. [17]. The difference is in a factor $\mathcal{E} /(\mathcal{E}-\omega)$, because in contrast to [17] the final photon mass squared $k_{\mu}^{2}=\omega^{2}\left(1-n^{2}\right)$ is not neglected for this Letter.

Substituting $n-1$ in Eq. (8) by the gravitational refractivity from Eq. (7) we can estimate how the quantum gravity would change the scattered photons' maximal energy $\omega_{m}$ (Compton edge, at $\theta=0$ ). The expected shift of the Compton edge is

$$
\omega_{m}(n)-\omega_{m}(1)=\frac{32 \gamma^{6} \omega_{0}^{2} \sin ^{4}\left(\theta_{0} / 2\right)}{(1+x)^{4}} \frac{\zeta}{M_{P}}
$$

relative to the vacuum $(n=1)$ kinematics. At sufficiently high $\gamma$, the huge value of $M_{P}$ is compensated, and the energy shift becomes detectable. Hence, this effect allows quantum-gravity induced space refractivity to be measured at accelerators by laser Compton scattering off high $\gamma$ electrons. For optical lasers and head-on collision the kinematic factor $x \approx 2 \times 10^{-5} \gamma$ and the right-hand side of Eq. (9) grows as $\gamma^{6}$ at $\mathrm{GeV}$ energies slowing down to $\gamma^{2}$ growth above $\mathrm{TeV}$ energies.

In order to probe space birefringence, one needs to measure the refractivity in Eq. (8) for scattered photons 
of opposite helicity. This may be achieved by exploring circularly polarized initial laser beams and helicity conservation. The polarization of the secondary photons in the case of scattering on unpolarized electrons is shown in Fig. 1, using formulas from Refs. [19,20]. At $\omega=\omega_{m}$ the polarization transfer is complete, such that the helicity of the Compton edge photons is fully defined by the laser light helicity. Consequently, in a birefringent vacuum the Compton edge energy is laser helicity dependent. Evaluating Eq. (8) for left and right helicity photons at $\theta=0$ yields

$$
\Delta n=n_{L}\left(\omega_{m}^{L}\right)-n_{R}\left(\omega_{m}^{R}\right)=\frac{(1+x)^{2}}{\gamma^{2}} A,
$$

where $\omega_{m}^{L}$ and $\omega_{m}^{R}$ are the highest energies for the Compton opposite helicity photons and $A=\left(\omega_{m}^{L}-\omega_{m}^{R}\right) /\left(\omega_{m}^{L}+\right.$ $\left.\omega_{m}^{R}\right)$ is an energy asymmetry.

Combining Eq. (10) with the gravitational birefringence from Eq. (4), we arrive to

$$
A=\frac{8 \gamma^{4} \omega_{0} \sin ^{2}\left(\theta_{0} / 2\right)}{(1+x)^{3}} \frac{\xi}{M_{P}},
$$

which proves that for sufficiently high values of $\gamma$ the Planck-scale space birefringence generates a measurable asymmetry. For lasers with $\omega_{0} \approx 3 \mathrm{eV}$ and $\theta_{0}=\pi, \gamma$ dependence of Eq. (11) changes from $\gamma^{4}$ to $\gamma$ in the $\mathrm{GeV}$ to $\mathrm{TeV}$ range.

Sensitivity of e $\gamma$ colliders. - To estimate sensitivity of the described methods we choose head-on collision $\left(\theta_{0}=\pi\right)$ of an ordinary green laser $\left(\omega_{0}=2.41 \mathrm{eV}\right)$ with two different energy electron beams, $6(x=0.22)$ and $250 \mathrm{GeV}(x=9.23)$. The first energy is available at

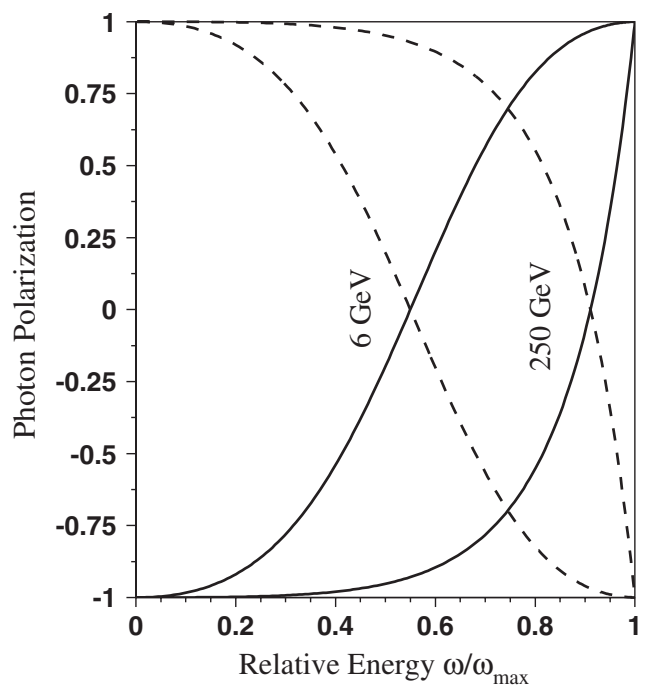

FIG. 1. Polarization of the Compton scattered photon on a 6 or $250 \mathrm{GeV}$ electron as a function of the photon energy. The solid and dotted lines correspond to the initial laser light helicity: +1 solid, -1 dotted. existent storage rings (e.g., PETRA-III [21]), while the higher energy may be accessible at the future International Linear Collider (ILC) [22]. A graphical view of the experimental reach is presented in Fig. 2. By using Eq. (11), the correspondence of the quantum-gravity scale $\left(\xi l_{P}=\xi / M_{P}\right)$ to the induced asymmetry is plotted. It is worth noting that asymmetries as small as $10^{-7}$ have been detected with a sensitivity $10^{-8}$ at the SLAC $50 \mathrm{GeV}$ experiments $[23,24]$, based on beam helicity flips. Thus, similar accuracies seem reachable at PETRA or at the ILC which suggests that the $6 \mathrm{GeV}$ machine could test space birefringence down to $10^{-31} \mathrm{~m}$ while the $250 \mathrm{GeV}$ machine reaches the Planck length.

The space refractivity measurement is more difficult, since one needs to detect an absolute energy shift which is relatively small. For that, the detector scale could be calibrated by the bremsstrahlung edge [17]. An alternative could be the measurement of the scattered electron momentum using a spectrometer. Exploring lasers of different wavelengths may give even better sensitivity. In either case the Compton edge detection is expected to be possible within an accuracy of $\Delta \omega_{m} / \omega_{m} \sim 10^{-3}$, thus probing space refractivity down to $10^{-27}$ and $10^{-31} \mathrm{~m}$ for the 6 and $250 \mathrm{GeV}$ machines, respectively.

As a candidate for a quantum-gravity signature, consider high energy photon speed anomaly observations reported in Ref. [17]. Refractivities $(1.69 \pm 0.47) \times 10^{-11}$ and

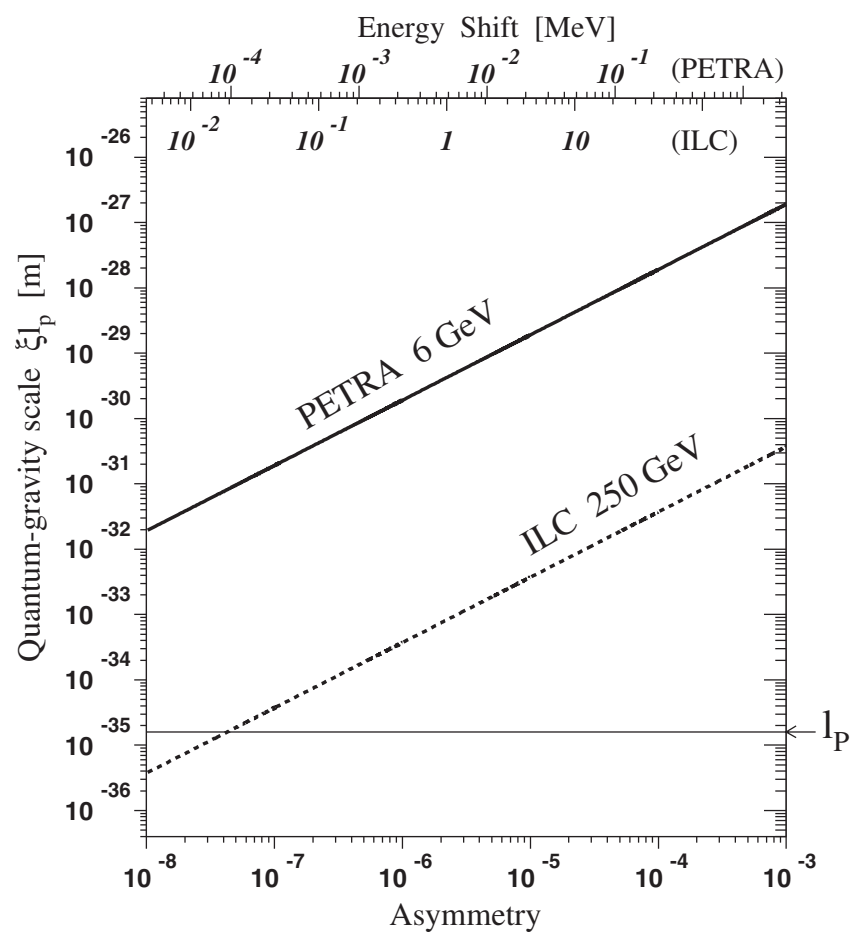

FIG. 2. Experimental reach of the accelerators for vacuum birefringence and refractivity. Birefringence at the scale $\xi$ will produce a Compton edge asymmetry (lower scale), while the refractivity produces absolute energy shifts (upper scale). The Planck length $l_{P}$ is shown by an arrow. 
$(4.07 \pm 0.05) \times 10^{-13}$ obtained with HERA $26.5 \mathrm{GeV}$ [25] and SLC $45.6 \mathrm{GeV}$ [26] electron beams may be interpreted as quantum-gravity manifestation at $(2.57 \pm 0.71) \times$ $10^{-28}$ and $(3.50 \pm 0.04) \times 10^{-30} \mathrm{~m}$ distances for the HERA and SLC measurements, respectively.

In contrast to the astrophysical methods, an accelerator Compton experiment is sensitive to the local properties of space at the laser-electron interaction point and along the scattered photon direction. Hence, space isotropy tests are also possible as the accelerator rotates together with Earth. For any preferred direction, the measured birefringence is expected to change as the scattered photon beam sweeps a circle over the celestial sphere. For a given direction $(\delta, \alpha)$ of the photon beam and a possible anisotropy axis $\left(\delta_{0}, \alpha_{0}\right)$, one expects

$$
\Delta n=\Delta n_{0}\left[\cos \delta \cos \delta_{0} \cos \left(\alpha-\alpha_{0}\right)+\sin \delta \sin \delta_{0}\right],
$$

where $\Delta n_{0}$ is the maximal birefringence, along the declination $\delta_{0}$ and right ascension $\alpha_{0}$. Despite the tight limits set by low energy high precision experiments on space anisotropy [27], the accelerator isotropy test is a valuable and complementary test at high energies.

In conclusion, an accelerator based experiment is proposed to probe space birefringence and refractivity close to the Planck scale in photon electron collisions.

I thank S. Schmitt for his careful and critical reading of the manuscript.

*vahagn.gharibyan@desy.de; http://desy.de/ vaagn

[1] C. Amsler et al., Phys. Lett. B 667, 1 (2008).

[2] G. Amelino-Camelia, J. R. Ellis, N. E. Mavromatos, D. V. Nanopoulos, and S. Sarkar, Nature (London) 393, 763 (1998).

[3] R. Gambini and J. Pullin, Phys. Rev. D 59, 124021 (1999).

[4] R. J. Gleiser, C. N. Kozameh, and F. Parisi, Classical Quantum Gravity 20, 4375 (2003).

[5] G. Gubitosi, G. Genovese, G. Amelino-Camelia, and A. Melchiorri, Phys. Rev. D 82, 024013 (2010).
[6] F. W. Hehl, P. Von Der Heyde, G. D. Kerlick, and J. M. Nester Rev. Mod. Phys. 48, 393 (1976).

[7] A. R. Prasanna and S. Mohanty, Gen. Relativ. Gravit. 41, 1905 (2009).

[8] R. C. Myers and M. Pospelov, Phys. Rev. Lett. 90, 211601 (2003).

[9] D. A. R. Dalvit, F. D. Mazzitelli and C. Molina-Paris, Phys. Rev. D 63, 084023 (2001).

[10] J. R. Ellis, N. E. Mavromatos, and D. V. Nanopoulos, Phys. Lett. B 665, 412 (2008).

[11] W. Dittrich and H. Gies, Phys. Rev. D 58, 025004 (1998).

[12] L. Bombelli and O. Winkler, Classical Quantum Gravity 21, L89 (2004).

[13] R. J. Gleiser and C. N. Kozameh, Phys. Rev. D 64, 083007 (2001).

[14] F. W. Stecker Astropart. Phys. 35, 95 (2011).

[15] S. McGlynn, D. J. Clark, A. J. Dean, L. Hanlon, S. McBreen, D. R. Willis, B. McBreen, A. J. Bird, and S. Foley, Astron. Astrophys. 466, 895 (2007).

[16] D. Heyman, S. Major, and F. Hinteleitner, Phys. Rev. D 69 , 105016 (2004).

[17] V. Gharibyan, Phys. Lett. B 611, 231 (2005).

[18] J. R. Ellis, N. E. Mavromatos, and D. V. Nanopoulos Phys. Lett. B 674, 83 (2009).

[19] F. W. Lipps and H. A. Tolhoek, Physica (Utrecht) 20, 85 (1954); 20, 385 (1954).

[20] W. H. McMaster, Rev. Mod. Phys. 33, 8 (1961).

[21] Report No. DESY-04-035, edited by K. Balewski, W. Brefeld, W. Decking, H. Franz, R. Rohlsberger, and E. Weckert.

[22] G. Aarons et al. (ILC Collaboration), arXiv:0709.1893.

[23] P.L. Anthony et al. (SLAC E158 Collaboration), Phys. Rev. Lett. 92, 181602 (2004).

[24] P. L. Anthony et al. (SLAC E158 Collaboration), Phys. Rev. Lett. 95, 081601 (2005).

[25] D. P. Barber et al., Nucl. Instrum. Methods Phys. Res., Sect. A 329, 79 (1993).

[26] G. Shapiro et al., in Proceedings of the 1993 Particle Accelerator Conference (PAC 93), Washington, DC (unpublished).

[27] D. Sudarsky, L. Urrutia, and H. Vucetich, Phys. Rev. Lett. 89, 231301 (2002). 